Supporting Information

Identification of Photoinitiators, Including Novel Phosphine Oxides, and Their Transformation Products in Food Packaging Materials and Indoor Dust in

\title{
Canada
}

Runzeng Liu* and Scott A. Mabury

Department of Chemistry, University of Toronto, 80 St. George Street, Toronto, M5S 3H6, Ontario Canada

*Corresponding author

Dr. Runzeng Liu

Department of Chemistry, University of Toronto

E-mail: $\underline{\text { rz.liu@utoronto.ca }}$

$+1(416) 946-7736$ 


\section{Table of Contents}

Materials

Instrumental Analysis

Table S1. Analyte names, abbreviations, and related information.

Table S2. Optimized MRM parameters in LC-MS/MS analysis.

Table S3. Validation and performance data of the developed method for food packaging materials.

Table S4. Validation and performance data of the developed method for dust matrix.

Table S5. Descriptive statistics of the measured PI concentrations $\left(\mathrm{ng} / \mathrm{dm}^{2}\right)$ in food packaging materials $(\mathrm{n}=33)$.

Table S6. Spearman correlation analysis of PIs in food packaging materials $(n=33)$.

Table S7. Estimated daily intakes of PIs (ng/kg bw/day) via dust ingestion.

Table S8. Descriptive statistics of the measured PI concentrations (ng/g) in different dust matrices.

Table S9. Spearman correlation analysis of PIs in indoor dust samples $(n=92)$.

Table S10. The starting concentrations and calculated half-life times $\left(t_{1 / 2}\right)$ of PIs in food packaging materials used for the photodegradation.

Table S11. Ratios between the measured concentrations of transformation products and parent chemicals.

Table S12. Human skin sensitization predicted by Pre-Skin.

Figure S1. Structures of the target BZPs, TXs, and ACIs in the present study.

Figure S2. Stability of PIs in food packaging materials in the dark $(n=3)$.

Figure S3. Concentrations of TX transformation products (mean \pm standard deviation, $\mathrm{n}=3$ ) in food packaging materials during the photodegradation experiments. 


\section{Materials}

Native standards of the PI analytes were obtained from TCI (Tokyo, Japan) and Combi-Blocks (San Diego, USA). Isotopically labeled benzophenone-d10 (BP-d10) and 2-isopropyl-d7-thioxanthone (2-ITX-d7) were purchased from Sigma Aldrich (Oakville, Canada), while triphenyl phosphate-d15 (TPHP-d15) was obtained from Cambridge Isotope Laboratories (Andover, USA). The purities of all target analytes were $>95 \%$. Stock solutions at concentrations of $1000 \mu \mathrm{g} / \mathrm{mL}$ were individually prepared in acetonitrile $(\mathrm{ACN})$, while intermediate solutions were prepared weekly from the stock solutions by methanol $(\mathrm{MeOH})$ dilution. HPLC-grade $\mathrm{ACN}$ and $\mathrm{MeOH}$ were obtained from Sigma Aldrich (Oakville, Canada). Formic acid (FA) was supplied by Caledon (Georgetown, Canada). Ultrapure water $\left(\mathrm{H}_{2} \mathrm{O}, 18.2 \mathrm{M} \Omega \times \mathrm{cm}\right)$ was generated by Purelab flex (Veolia Water Technologies, Mississauga, Canada). Adsorbent materials, including primary secondary amine (PSA) and octadecyl silane (C18), were purchased from Agilent Technologies (Santa Clara, USA).

\section{Instrumental Analysis}

The quantification of the targets was performed on a Waters ultrahigh performance liquid chromatograph coupled to a Xevo triple-quadrupole mass spectrometer (Milford, USA). Electrospray ionization (ESI) was operated in the positive mode. The cone and desolvation gas flow were 120 and $800 \mathrm{~L} / \mathrm{h}$, respectively. The source temperature was set to $120^{\circ} \mathrm{C}$, and the desolvation temperature was $400^{\circ} \mathrm{C}$. Detailed information on the multiple reaction monitoring parameters is presented in the supporting information (Table S2). A Waters ACQUITY BEH C18 analytical column $(2.1 \times 100 \mathrm{~mm}, 1.7 \mu \mathrm{m})$ was used for the analyte separation, with a flow rate of 0.3 $\mathrm{mL} / \mathrm{min}$. The column temperature was set to $60^{\circ} \mathrm{C}$. The flow gradient was started at a composition of 40:60 $\left(\mathrm{MeOH} / \mathrm{H}_{2} \mathrm{O}, \mathrm{v} / \mathrm{v}, 0.1 \% \mathrm{FA}\right.$ additive in each phase). It was held steady for $3 \mathrm{~min}$, linearly ramped to $100 \% \mathrm{MeOH}$ over $7 \mathrm{~min}$, and then held steady for another $5 \mathrm{~min}$, for a total analysis time of $15 \mathrm{~min}$. 
Table S1. Analyte names, abbreviations, and related information.

\begin{tabular}{|c|c|c|c|c|c|c|}
\hline \multirow{2}{*}{ Analyte name } & \multirow{2}{*}{ Abbreviation } & \multirow{2}{*}{ CAS } & \multicolumn{2}{|c|}{ Production and imported volume } & \multirow{2}{*}{$\begin{array}{l}\log \\
\mathrm{K}_{\mathrm{ow}}\end{array}$} & \multirow{2}{*}{$\begin{array}{l}\text { Log } \\
\mathrm{K}_{\mathrm{oa}}\end{array}$} \\
\hline & & & Canada $(\mathrm{kg})$ & US (lbs) & & \\
\hline Benzophenone & $\mathrm{BP}$ & $119-61-9$ & $\begin{array}{c}35000- \\
135000\end{array}$ & $3,870,000$ & 3.18 & 7.28 \\
\hline 1,2-Diphenyl-1,2-ethanedione & Benzil & $134-81-6$ & NA & NA & 3.38 & 9.88 \\
\hline 1-Hydroxycyclohexylphenylketone & PI-184 & $947-19-3$ & NA & $1,210,000$ & 2.44 & 6.38 \\
\hline 4-Methylbenzophenone & 4-MBP & $134-84-9$ & NA & withheld & 3.69 & 7.75 \\
\hline 2-Ethylanthraquinone & EAQ & $84-51-5$ & NA & withheld & 4.37 & 11.1 \\
\hline 4-Phenylbenzophenone & PBZ & $2128-93-0$ & NA & $25,000-100,000$ & 4.91 & 10.1 \\
\hline 2,2-Dimethoxy-2-phenylacetophenone & PI-651 & $24650-42-8$ & NA & 903,000 & 2.95 & 2.95 \\
\hline Methyl-2-(benzoyl)benzoate & MBB & $606-28-0$ & NA & withheld & 2.70 & 8.99 \\
\hline $\begin{array}{l}\text { 4,4'-Bis(dimethylamino)benzophenone } \\
\text { (Michler's ketone) }\end{array}$ & MK & $90-94-8$ & $1000-10000 \mathrm{~kg}$ & NA & 3.87 & 11.6 \\
\hline $\begin{array}{l}\text { 4,4'-Bis(diethylamino)benzophenone } \\
\text { (Michler's ethylketone) }\end{array}$ & MEK & $90-93-7$ & NA & withheld & 5.47 & 12.7 \\
\hline Ethyl-4-dimethylaminobenzoate & EDMAB & $10287-53-3$ & NA & withheld & 2.89 & 7.41 \\
\hline 2-Ethylhexyl-4-(dimethylamino)benzoate & EHDAB & $21245-02-3$ & NA & withheld & 5.77 & 9.56 \\
\hline 2-Benzyl-2-(dimethylamino)-4'-morpholinobutyrophenone & PI-369 & $119313-12-1$ & NA & $100,000-500,000$ & 4.50 & 14.6 \\
\hline 2-Methyl-4'-(methylthio)-2-morpholinopropiophenone & PI-907 & $71868-10-5$ & NA & 523000 & 2.73 & 11.6 \\
\hline
\end{tabular}




\begin{tabular}{|c|c|c|c|c|c|c|}
\hline 4-(Dimethylamino)benzophenone & DMAB & $530-44-9$ & NA & NA & 3.32 & 9.22 \\
\hline 4,4'-Diaminobenzophenone & DABP & $611-98-3$ & NA & NA & 1.31 & 12.3 \\
\hline Ethyl-4-aminobenzoate & $\mathrm{EAB}$ & $94-09-7$ & NA & withheld & 1.86 & 8.04 \\
\hline Thioxanthone & $\mathrm{TX}$ & $492-22-8$ & NA & NA & 3.99 & 9.67 \\
\hline 2-Isopropylthioxanthone & 2-ITX & $5495-84-1$ & NA & 172,000 & 5.54 & 10.9 \\
\hline 2,4-Diethylthioxanthone & DETX & $82799-44-8$ & NA & withheld & 6.16 & 11.5 \\
\hline 2-Chlorothioxanthone & 2-Cl-TX & $86-39-5$ & NA & NA & 4.73 & 11.8 \\
\hline 2-isopropyl-10-oxothioxanthen-9-one & 2-ITX-O & $\mathrm{NA}^{\mathrm{a}}$ & NA & NA & 3.32 & 12.9 \\
\hline 2-diisopropyl-10,10-dioxothioxanthen-9-one & 2-ITX-O ${ }_{2}$ & $\mathrm{NA}^{\mathrm{a}}$ & NA & NA & 3.86 & 11.3 \\
\hline 2,4-diethyl-10-oxothioxanthen-9-one & DETX-O & $591773-88-5$ & NA & NA & 3.94 & 13.5 \\
\hline 2,4-diethyl-10,10-dioxothioxanthen-9-one & DETX-O ${ }_{2}$ & $82799-45-9$ & NA & NA & 4.48 & 11.9 \\
\hline Triphenylphosphine oxide & ТРPO & $791-28-6$ & NA & withheld & 2.87 & 10.5 \\
\hline 2,4,6-Trimethylbenzoyldiphenylphosphine oxide & TPO & $75980-60-8$ & NA & 550,000 & 3.87 & 12.3 \\
\hline Ethylphenyl(2,4,6-trimethylbenzoyl)phosphinate & TPO-L & $84434-11-7$ & NA & NA & 3.13 & 10.6 \\
\hline Phenylbis(2,4,6-trimethylbenzoyl)phosphine oxide & PI-819 & $162881-26-7$ & NA & withheld & 4.65 & 13.9 \\
\hline
\end{tabular}

NA: not available. Production and imported volume were from Environment and Climate Change Canada and US EPA. LogK ${ }_{\text {ow }}$ and LogK were calculated by EPI Suite 4.1. 
Table S2. Optimized MRM parameters in LC-MS/MS analysis.

\begin{tabular}{|c|c|c|c|c|}
\hline Compounds & $\begin{array}{l}\text { Precursor ion } \\
\qquad(m / z)\end{array}$ & $\begin{array}{l}\text { Product ion } \\
\qquad(\mathrm{m} / \mathrm{z})\end{array}$ & $\begin{array}{c}\text { Cone } \\
\text { potential (V) }\end{array}$ & $\begin{array}{c}\text { Collision } \\
\text { energy }(\mathrm{V})\end{array}$ \\
\hline \multirow[t]{2}{*}{ BP } & \multirow[t]{2}{*}{183.1} & 105.1 & \multirow{2}{*}{128} & 22 \\
\hline & & 77.1 & & 12 \\
\hline \multirow[t]{2}{*}{ Benzil } & \multirow[t]{2}{*}{211.2} & 77.1 & \multirow[t]{2}{*}{28} & 26 \\
\hline & & 105.1 & & 8 \\
\hline \multirow[t]{2}{*}{ PI-184 } & \multirow[t]{2}{*}{187.1} & 105.1 & \multirow[t]{2}{*}{32} & 28 \\
\hline & & 77.1 & & 14 \\
\hline \multirow[t]{2}{*}{ 4-MBP } & \multirow[t]{2}{*}{197.2} & 105.1 & \multirow[t]{2}{*}{36} & 14 \\
\hline & & 119.1 & & 16 \\
\hline \multirow[t]{2}{*}{ EAQ } & \multirow[t]{2}{*}{237.1} & 152.2 & \multirow[t]{2}{*}{32} & 34 \\
\hline & & 209.1 & & 20 \\
\hline \multirow[t]{2}{*}{ PBZ } & \multirow[t]{2}{*}{259.2} & 77.2 & \multirow[t]{2}{*}{40} & 36 \\
\hline & & 105.1 & & 16 \\
\hline \multirow[t]{2}{*}{ PI-651 } & \multirow[t]{2}{*}{225.2} & 105.1 & \multirow[t]{2}{*}{68} & 20 \\
\hline & & 197.1 & & 12 \\
\hline MBB & \multirow[t]{2}{*}{241.1} & 152.2 & \multirow[t]{2}{*}{20} & 34 \\
\hline$\ldots \ldots$ & & 209.1 & & 16 \\
\hline \multirow[t]{2}{*}{ MK } & \multirow[t]{2}{*}{269.3} & 148.1 & \multirow[t]{2}{*}{88} & 18 \\
\hline & & 77.2 & & 52 \\
\hline \multirow[t]{2}{*}{ MEK } & \multirow[t]{2}{*}{325.3} & 176.2 & \multirow{2}{*}{6} & 20 \\
\hline & & 133.0 & & 44 \\
\hline \multirow[t]{2}{*}{ EDMAB } & \multirow[t]{2}{*}{194.2} & 151.0 & 52 & 24 \\
\hline & & 166.1 & & 18 \\
\hline EHDAB & 278.2 & 166.2 & 48 & 20 \\
\hline & & 151.0 & & 30 \\
\hline PI-369 & 367.2 & 176.2 & 68 & 12 \\
\hline & & 190.2 & & 26 \\
\hline PI-907 & 280.2 & 165.1 & 8 & 16 \\
\hline & & 117.4 & & 32 \\
\hline
\end{tabular}




\begin{tabular}{|c|c|c|c|c|}
\hline \multirow[t]{2}{*}{ DMAB } & \multirow[t]{2}{*}{226.2} & 105.1 & \multirow{2}{*}{14} & 18 \\
\hline & & 77.1 & & 30 \\
\hline \multirow[t]{2}{*}{ EAB } & \multirow[t]{2}{*}{166.2} & 94.2 & \multirow[t]{2}{*}{32} & 18 \\
\hline & & 138.1 & & 10 \\
\hline \multirow[t]{2}{*}{$\mathrm{TX}$} & \multirow[t]{2}{*}{213.1} & 184.1 & \multirow{2}{*}{36} & 28 \\
\hline & & 152.1 & & 38 \\
\hline \multirow[t]{2}{*}{ 2-ITX } & \multirow[t]{2}{*}{255.2} & 213.1 & \multirow[t]{2}{*}{42} & 20 \\
\hline & & 184.1 & & 38 \\
\hline \multirow[t]{2}{*}{ DETX } & \multirow[t]{2}{*}{269.2} & 241.0 & \multirow[t]{2}{*}{52} & 22 \\
\hline & & 212.9 & & 30 \\
\hline \multirow[t]{2}{*}{ 2-Cl-TX } & \multirow[t]{2}{*}{247.0} & 184.0 & \multirow{2}{*}{100} & 34 \\
\hline & & 212.1 & & 26 \\
\hline \multirow[t]{2}{*}{ BP-d10 } & \multirow[t]{2}{*}{193.2} & 82.0 & \multirow[t]{2}{*}{18} & 16 \\
\hline & & 109.9 & & 39 \\
\hline \multirow[t]{2}{*}{ 2-ITX-d7 } & \multirow[t]{2}{*}{262.2} & 214.2 & \multirow[t]{2}{*}{38} & 22 \\
\hline & & 185.2 & & 38 \\
\hline \multirow[t]{2}{*}{ 2-ITX-O } & \multirow[t]{2}{*}{271.1} & 239.1 & \multirow[t]{2}{*}{20} & 26 \\
\hline & & 254.1 & & 16 \\
\hline \multirow{2}{*}{$2-\mathrm{ITX}-\mathrm{O}_{2}$} & \multirow[t]{2}{*}{287.1} & 181.2 & \multirow[t]{2}{*}{46} & 20 \\
\hline & & 245.2 & & 16 \\
\hline \multirow[t]{2}{*}{ DETX-O } & 285.2 & 253.2 & 46 & 30 \\
\hline & & 268.2 & & 16 \\
\hline DETX-O ${ }_{2}$ & 301.2 & 165.2 & 46 & 40 \\
\hline & & 273.2 & & 16 \\
\hline ТРPO & 279.2 & 77.1 & 48 & 42 \\
\hline & & 173.2 & & 34 \\
\hline TPO & 371.1 & 119.2 & 64 & 46 \\
\hline & & 147.1 & & 30 \\
\hline TPO-L & 317.2 & 91.2 & 4 & 56 \\
\hline & & 119.2 & & 42 \\
\hline PI-819 & 441.1 & 119.2 & 86 & 46 \\
\hline & & 147.1 & & 24 \\
\hline
\end{tabular}


Table S3. Validation and performance data of the developed method for food packaging materials.

\begin{tabular}{|c|c|c|c|c|c|c|c|c|}
\hline \multirow[b]{2}{*}{ Compounds } & \multicolumn{3}{|c|}{ Low spiking level (10 ng/g) } & \multicolumn{3}{|c|}{ Low spiking level $(100$ ng/g) } & \multirow[b]{2}{*}{$\begin{array}{c}\text { MQL based on } \\
\text { area }\left(\mathrm{ng} / \mathrm{dm}^{2}\right)\end{array}$} & \multirow[b]{2}{*}{$\begin{array}{l}\text { MQL based on } \\
\text { weight }(\mathrm{ng} / \mathrm{g})\end{array}$} \\
\hline & $\begin{array}{c}\text { Recovery } \\
(\%)\end{array}$ & $\begin{array}{c}\mathrm{RSD} \\
(\%)\end{array}$ & $\begin{array}{c}\text { Matrix effect } \\
(\%)\end{array}$ & $\begin{array}{c}\text { Recovery } \\
(\%)\end{array}$ & $\begin{array}{c}\mathrm{RSD} \\
(\%)\end{array}$ & $\begin{array}{c}\text { Matrix } \\
\text { effect } \\
(\%)\end{array}$ & & \\
\hline BP & 94.0 & 12.1 & 95.0 & 96.9 & 12.4 & 95.4 & 1.0 & 0.3 \\
\hline Benzil & 93.1 & 7.2 & 101 & 80.0 & 3.4 & 90.6 & 1.6 & 0.4 \\
\hline PI-184 & 90.5 & 12.4 & 92.9 & 103 & 7.5 & 98.7 & 2.0 & 0.5 \\
\hline EAQ & 85.5 & 14.7 & 95.9 & 96.0 & 6.6 & 95.7 & 6.2 & 1.5 \\
\hline PBZ & 98.0 & 5.8 & 101 & 94.9 & 10.1 & 93.3 & 0.6 & 0.2 \\
\hline PI-651 & 95.1 & 12.4 & 95.3 & 97.5 & 8.5 & 95.1 & 0.8 & 0.2 \\
\hline MBB & 85.5 & 7.2 & 95.9 & 89.7 & 6.2 & 98.8 & 0.8 & 0.2 \\
\hline EDMAB & 89.2 & 4.3 & 92.9 & 96.1 & 4.7 & 105 & 0.2 & 0.04 \\
\hline EHDAB & 97.7 & 5.5 & 96.3 & 101 & 7.9 & 94.3 & 0.1 & 0.03 \\
\hline PI-369 & 92.3 & 5.9 & 106 & 87.3 & 8.2 & 109 & 0.8 & 0.2 \\
\hline PI-907 & 98.3 & 2.1 & 101 & 89.7 & 3.9 & 99.7 & 1.1 & 0.3 \\
\hline DABP & 73.0 & 3.4 & 83.2 & 68.0 & 6.8 & 81.8 & 0.2 & 0.06 \\
\hline
\end{tabular}




\begin{tabular}{|c|c|c|c|c|c|c|c|c|}
\hline DMAB & 95.0 & 1.1 & 100 & 97.6 & 3.8 & 80.5 & 0.2 & 0.04 \\
\hline $\mathrm{TX}$ & 94.0 & 5.2 & 93.6 & 87.1 & 9.1 & 101 & 2.4 & 0.6 \\
\hline 2-ITX & 95.3 & 0.4 & 99.9 & 97.7 & 3.0 & 96.2 & 0.1 & 0.03 \\
\hline 2-Cl-TX & 95.7 & 14.8 & 93.0 & 85.4 & 4.5 & 90.3 & 0.4 & 0.1 \\
\hline DETX & 95.3 & 4.7 & 96.9 & 87.6 & 5.5 & 96.0 & 0.1 & 0.03 \\
\hline $2-\mathrm{ITX}-\mathrm{O}_{2}$ & 90.7 & 3.7 & 101 & 99.3 & 7.1 & 102 & 2.6 & 0.7 \\
\hline DETX-O & 98.7 & 2.8 & 99.5 & 98.6 & 9.2 & 100 & 0.2 & 0.04 \\
\hline DETX-O ${ }_{2}$ & 99.3 & 4.7 & 106 & 97.6 & 12.6 & 107 & 1.8 & 0.44 \\
\hline ТРPO & 88.0 & 11.7 & 99.1 & 87.4 & 4.6 & 86.2 & 4.0 & 1.0 \\
\hline PI-819 & 62.8 & 3.1 & 93.1 & 63.2 & 3.4 & 89.7 & 4.8 & 1.2 \\
\hline BP-d10 & 98.3 & 7.2 & 95.9 & 91.8 & 3.4 & 92.3 & - & - \\
\hline 2-ITX-d7 & 90.8 & 7.2 & 99.1 & 95.0 & 7.1 & 96.8 & - & - \\
\hline TPHP-d15 & 93.9 & 6.6 & 98.4 & 92.4 & 6.0 & 95.5 & - & - \\
\hline
\end{tabular}


Table S4. Validation and performance data of the developed method for dust matrix.

\begin{tabular}{|c|c|c|c|c|c|c|c|}
\hline \multirow[b]{2}{*}{ Compounds } & \multicolumn{3}{|c|}{ Low spiking level (10 ng/g) } & \multicolumn{3}{|c|}{ High spiking level (100 ng/g) } & \multirow[b]{2}{*}{ MQL (ng/g) } \\
\hline & $\begin{array}{c}\text { Recovery } \\
(\%)\end{array}$ & $\begin{array}{l}\text { RSD } \\
(\%)\end{array}$ & $\begin{array}{c}\text { Matrix effect } \\
(\%)\end{array}$ & Recovery (\%) & $\begin{array}{c}\mathrm{RSD} \\
(\%)\end{array}$ & $\begin{array}{c}\text { Matrix effect } \\
(\%)\end{array}$ & \\
\hline $\mathrm{BP}$ & 95.7 & 13.3 & 100 & 93.8 & 5.4 & 97.0 & 1.0 \\
\hline Benzil & 98.3 & 5.8 & 96.1 & 95.9 & 6.8 & 96.4 & 1.9 \\
\hline PI-184 & 94.9 & 1.8 & 98.2 & 91.5 & 6.4 & 103 & 2.0 \\
\hline PBZ & 89.8 & 5.9 & 90.6 & 69.7 & 2.9 & 79.3 & 0.2 \\
\hline PI-651 & 96.5 & 8.8 & 97.7 & 74.2 & 6.1 & 80.5 & 0.9 \\
\hline MBB & 98.6 & 3.6 & 116 & 82.1 & 7.6 & 106 & 0.7 \\
\hline EHDAB & 81.1 & 4.6 & 86.6 & 80.5 & 4.1 & 82.0 & 0.2 \\
\hline PI-369 & 80.7 & 2.6 & 90.6 & 76.3 & 4.0 & 109 & 0.8 \\
\hline PI-907 & 103 & 2.0 & 98.8 & 114 & 3.8 & 116 & 1.0 \\
\hline DABP & 75.7 & 2.5 & 75.5 & 53.5 & 8.8 & 98 & 0.3 \\
\hline
\end{tabular}




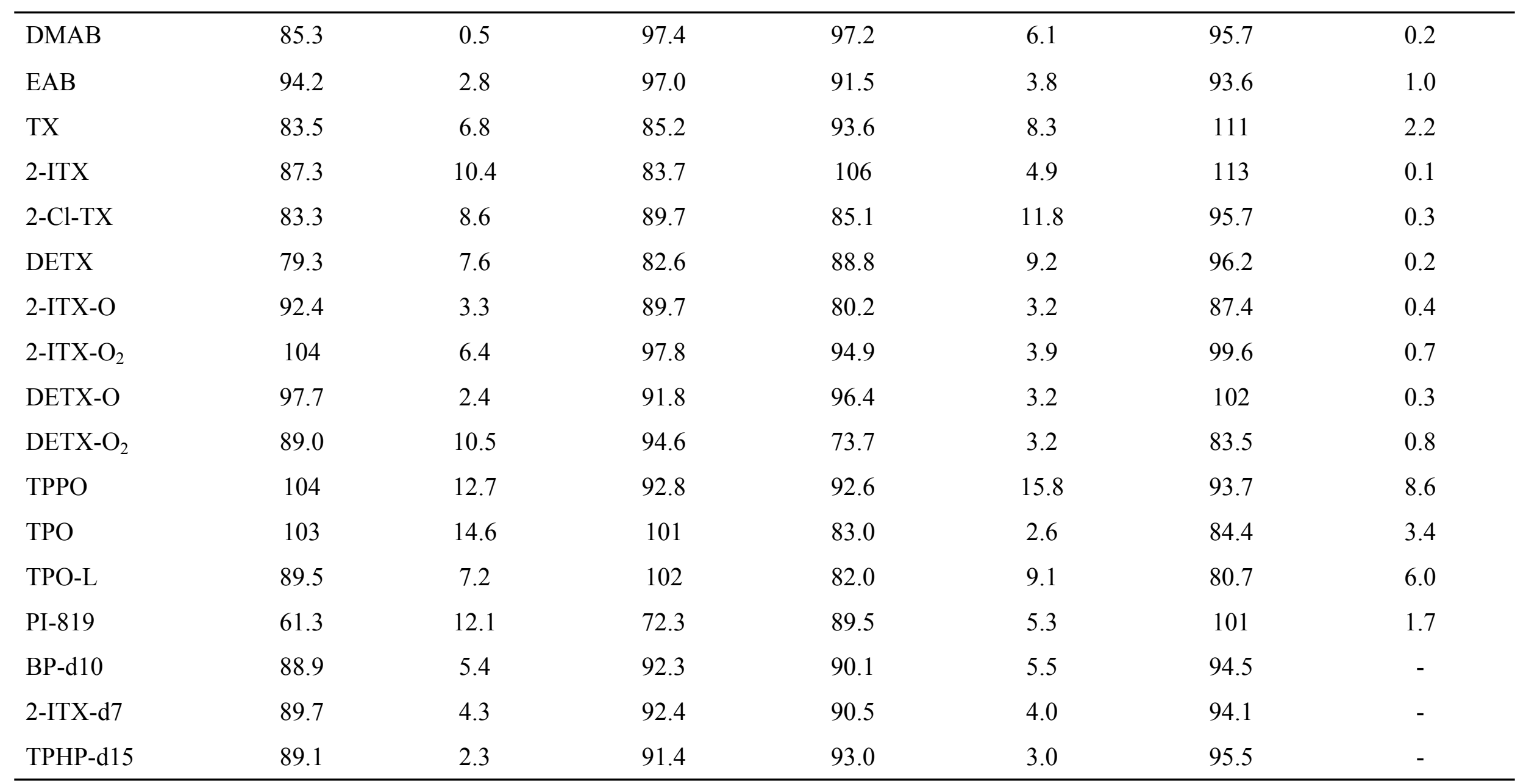


Table S5. Descriptive statistics of the measured PI concentrations $\left(\mathrm{ng} / \mathrm{dm}^{2}\right)$ in food packaging materials $(n=33)$.

\begin{tabular}{|c|c|c|c|}
\hline Compounds & $\mathrm{GM}^{\mathrm{a}}$ & median & Range \\
\hline $\mathrm{BP}$ & 5101 & 7866 & $205-21007$ \\
\hline PI-651 & 370 & 401 & $12.4-10023$ \\
\hline 4-MBP & 107 & 106 & $3.89-1776$ \\
\hline MBB & 513 & 559 & $8.33-14774$ \\
\hline PBZ & 72.1 & 126 & $1.57-2191$ \\
\hline PI-184 & 655 & 614 & $19.7-33003$ \\
\hline Benzil & 27.0 & 40.5 & $<\mathrm{MQL}-310$ \\
\hline EAQ & 4.53 & $<\mathrm{MQL}$ & $<\mathrm{MQL}-15.1$ \\
\hline$\sum \mathrm{BZPs}$ & 8020 & 11151 & $275-59255$ \\
\hline MK & 121 & 216 & $<\mathrm{MQL}-1478$ \\
\hline MEK & 278 & 376 & $2.34-4789$ \\
\hline EAB & 7.19 & 6.75 & $<\mathrm{MQL}-1507$ \\
\hline EDMAB & 190 & 224 & $2.64-16061$ \\
\hline EHDAB & 17.8 & 16.2 & $<\mathrm{MQL}-1223$ \\
\hline PI-369 & 144 & 83.9 & $6.09-31767$ \\
\hline PI-907 & 81.3 & 127 & $<\mathrm{MQL}-8257$ \\
\hline DABP & 2.10 & 2.36 & $<\mathrm{MQL}-29.0$ \\
\hline DMAB & 16.6 & 40.0 & $<\mathrm{MQL}-152$ \\
\hline$\sum \mathrm{ACIs}$ & 1270 & 1422 & $40.1-55401$ \\
\hline $\mathrm{TX}$ & 1.81 & $<\mathrm{MQL}$ & $<\mathrm{MQL}-6.78$ \\
\hline 2-ITX & 230 & 389 & $2.71-6358$ \\
\hline DETX & 61.6 & 116 & $<\mathrm{MQL}-2174$ \\
\hline 2-Cl-TX & $<\mathrm{MQL}$ & $<\mathrm{MQL}$ & $<\mathrm{MQL}$ \\
\hline$\sum \mathrm{TXs}$ & 350 & 610 & $6.92-6671$ \\
\hline ТРPO & 87.3 & 99.2 & $<\mathrm{MQL}-2574$ \\
\hline ТРО & 210 & 332 & $<\mathrm{MQL}-4896$ \\
\hline TPO-L & 75.2 & 65.7 & $<\mathrm{MQL}-222141$ \\
\hline PI-819 & 16.3 & 25.0 & $<\mathrm{MQL}-243$ \\
\hline$\sum \mathrm{POs}$ & 599 & 703 & $<\mathrm{MQL}-2383$ \\
\hline$\sum$ PIs & 10976 & 14550 & $382-112975$ \\
\hline
\end{tabular}


Table S6. Spearman correlation analysis of PIs in food packaging materials $(n=33)$.

\begin{tabular}{|c|c|c|c|c|c|c|c|c|c|c|c|c|c|c|c|c|c|c|c|c|c|c|}
\hline & MEK & PI-907 & EHDAB & MK & DMAB & EDMAB & EAB & DABP & PI-369 & PBZ & MBB & Benzil & PI-184 & BP & PI-651 & MBP & TPO-L & PI-819 & TPO & ТPPO & 2-ITX & DETX \\
\hline MEK & 1 & & & & & & & & & & & & & & & & & & & & & \\
\hline PI-907 & $.788^{* *}$ & 1 & & & & & & & & & & & & & & & & & & & & \\
\hline EHDAB & $.670 * *$ & $.388^{* *}$ & 1 & & & & & & & & & & & & & & & & & & & \\
\hline MK & $.614^{* *}$ & $.631^{* *}$ & $.471 * *$ & 1 & & & & & & & & & & & & & & & & & & \\
\hline DMAB & $.511^{* *}$ & $.567 * *$ & $.544 * *$ & $.611 * *$ & 1 & & & & & & & & & & & & & & & & & \\
\hline EDMAB & $.705^{* *}$ & $.627^{* *}$ & $.718^{* *}$ & $.583^{* *}$ & $.480^{* *}$ & 1 & & & & & & & & & & & & & & & & \\
\hline EAB & $.472 * *$ & $.443 * *$ & $.507 * *$ & .313 & .277 & $.665^{* *}$ & 1 & & & & & & & & & & & & & & & \\
\hline DABP & $.756^{* *}$ & $.694 * *$ & $.631 * *$ & $.688^{* * *}$ & $.466^{* *}$ & $.708^{* *}$ & $.673 * *$ & 1 & & & & & & & & & & & & & & \\
\hline PI-369 & $.715^{* *}$ & $.471^{* *}$ & $.818^{* *}$ & $.571 * *$ & $.583^{* *}$ & $.788^{* * *}$ & $.605^{* *}$ & $.673^{* *}$ & 1 & & & & & & & & & & & & & \\
\hline PBZ & $.543 * *$ & $.421^{* *}$ & $.768^{* *}$ & $.562 * *$ & $.682^{* *}$ & $.693 * *$ & $.439 *$ & $.484 * *$ & $.604 * *$ & 1 & & & & & & & & & & & & \\
\hline MBB & $.533^{* *}$ & $.435^{*}$ & $.689 * *$ & $.548^{* *}$ & $.642^{* *}$ & $.644 * *$ & $.443 * *$ & $.490^{* *}$ & $.729^{* *}$ & $.741^{* *}$ & 1 & & & & & & & & & & & \\
\hline Benzil & $.422^{*}$ & $.562^{* *}$ & $.423 *$ & .177 & $.456^{* *}$ & $.502 * *$ & $.596 * *$ & $.394 *$ & $.376^{*}$ & $.488^{* *}$ & $.405 *$ & 1 & & & & & & & & & & \\
\hline PI-184 & $.593 * *$ & $.601 * *$ & $.606^{* *}$ & $.518^{* *}$ & $.476^{* *}$ & $.591^{* *}$ & $.542 * *$ & $.750^{* *}$ & $.688^{* *}$ & $.379^{*}$ & $.627^{* *}$ & $.408^{*}$ & 1 & & & & & & & & & \\
\hline BP & $.610^{* *}$ & $.435^{*}$ & $.828^{* *}$ & $.591 * *$ & $.697^{* *}$ & $.679 * *$ & $.465 * *$ & $.541^{* *}$ & $.747^{* *}$ & $.885^{* *}$ & $.833^{* *}$ & $.434 *$ & $.522^{* *}$ & 1 & & & & & & & & \\
\hline PI-651 & $.599 * *$ & $.609 * *$ & $.516^{* *}$ & $.747^{* *}$ & $.635^{* *}$ & $.495^{* *}$ & .269 & $.694 * *$ & $.599^{* *}$ & $.447^{* *}$ & $.571^{* *}$ & .273 & $.648^{* *}$ & $.531^{* *}$ & 1 & & & & & & & \\
\hline MBP & $.623^{* *}$ & $.620^{* *}$ & $.405^{*}$ & $.483 * *$ & .251 & $.510 * *$ & $.479 * *$ & $.468^{* *}$ & .319 & $.577^{* *}$ & $.384 *$ & $.537^{* *}$ & $.355^{*}$ & $.522^{* *}$ & .306 & 1 & & & & & & \\
\hline TPO-L & $.561^{* *}$ & $.416^{*}$ & $.730^{* *}$ & $.366^{*}$ & $.414^{*}$ & $.651^{* *}$ & $.496 * *$ & $.530^{* *}$ & $.716^{* *}$ & $.512^{* *}$ & $.630^{* *}$ & .254 & $.598^{* *}$ & $.559^{* *}$ & $.513^{* *}$ & .181 & 1 & & & & & \\
\hline PI-819 & $.448^{* *}$ & .269 & $.458^{* *}$ & $.647^{* *}$ & $.453^{* *}$ & $.485 * *$ & .151 & $.464 * *$ & $.521^{* *}$ & $.553^{* *}$ & $.491^{* *}$ & -.040 & $.421^{*}$ & $.524 * *$ & $.554^{* *}$ & $.356^{*}$ & .275 & 1 & & & & \\
\hline TPO & $.784 * *$ & $.581^{* *}$ & $.724 * *$ & $.746 * *$ & $.685^{* *}$ & $.644 * *$ & .304 & $.731^{* *}$ & $.764 * *$ & $.576^{* *}$ & $.575^{* *}$ & .299 & $.671^{* *}$ & $.632^{* *}$ & $.774 * *$ & .324 & $.577^{* *}$ & $.607 * *$ & 1 & & & \\
\hline ТPPO & $.694 * *$ & $.613 * *$ & $.697^{* *}$ & $.673^{* *}$ & $.613^{* *}$ & $.723 * *$ & $.555^{* *}$ & $.750^{* *}$ & $.784 * *$ & $.559 * *$ & $.710^{* *}$ & $.361^{*}$ & $.708^{* *}$ & $.782^{* *}$ & $.660^{* *}$ & $.424 *$ & $.529 * *$ & $.497 * *$ & $.677^{* *}$ & 1 & & \\
\hline 2-ITX & $.719 * *$ & $.590^{* *}$ & $.785 * *$ & $.497 * *$ & $.551^{* *}$ & $.796 * *$ & $.713^{* *}$ & $.833^{* *}$ & $.768 * *$ & $.621^{* *}$ & $.631^{* *}$ & $.421^{*}$ & $.613^{* *}$ & $.685^{* *}$ & $.571^{* *}$ & $.361^{*}$ & $.751^{* *}$ & $.365^{*}$ & $.621^{* *}$ & $.772^{* *}$ & 1 & \\
\hline DETX & $.760 * *$ & $.608^{* *}$ & $.613 * *$ & $.769 * *$ & $.585^{* *}$ & $.683 * *$ & $.384^{*}$ & $.710^{* *}$ & $.690 * *$ & $.643^{* *}$ & $.521^{* *}$ & .184 & $.591 * *$ & $.662^{* *}$ & $.671 * *$ & $.611^{* *}$ & $.486^{* *}$ & $.714 * *$ & $.795^{* *}$ & $.659^{* *}$ & $.599 *$ & 1 \\
\hline
\end{tabular}


Table S7. Estimated daily intakes of PIs (ng/kg bw/day) via dust ingestion

\begin{tabular}{|c|c|c|c|c|}
\hline \multirow{2}{*}{ Compounds } & \multicolumn{2}{|c|}{ GM } & \multicolumn{2}{|c|}{$95^{\text {th }}$} \\
\hline & Toddlers & Adults & Toddlers & Adults \\
\hline $\mathrm{BP}$ & 3.90 & 0.32 & 13.4 & 1.09 \\
\hline Benzil & 0.33 & 0.03 & 3.52 & 0.29 \\
\hline PI-184 & 0.45 & 0.037 & 4.28 & 0.35 \\
\hline PI-651 & 0.09 & 0.008 & 1.14 & 0.09 \\
\hline 4-MBP & 0.07 & 0.008 & 0.41 & 0.03 \\
\hline MBB & 0.14 & 0.01 & 1.64 & 0.13 \\
\hline PBZ & 0.06 & 0.005 & 0.46 & 0.04 \\
\hline EAQ & 0.04 & 0.003 & 0.04 & 0.003 \\
\hline$\sum \mathrm{BZPs}$ & 6.57 & 0.53 & 33.7 & 2.74 \\
\hline $\mathrm{MK}$ & 0.04 & 0.003 & 0.32 & 0.03 \\
\hline MEK & 0.05 & 0.004 & 0.28 & 0.02 \\
\hline EAB & 0.07 & 0.006 & 1.00 & 0.08 \\
\hline EDMAB & 0.03 & 0.002 & 0.27 & 0.02 \\
\hline EHDAB & 0.05 & 0.004 & 0.52 & 0.05 \\
\hline PI-369 & 0.007 & 0.001 & 0.23 & 0.02 \\
\hline PI-907 & 0.02 & 0.002 & 0.56 & 0.05 \\
\hline DABP & 0.01 & 0.001 & 0.12 & 0.01 \\
\hline DMAB & 0.005 & 0.004 & 0.28 & 0.02 \\
\hline$\sum$ ACIs & 0.63 & 0.05 & 3.61 & 0.29 \\
\hline $\mathrm{TX}$ & 0.01 & 0.001 & 0.03 & 0.003 \\
\hline 2-ITX & 0.12 & 0.01 & 0.83 & 0.07 \\
\hline DETX & 0.02 & 0.002 & 0.70 & 0.06 \\
\hline$\sum \mathrm{TXs}$ & 0.23 & 0.02 & 1.50 & 0.12 \\
\hline ТРРО & 1.34 & 0.11 & 24.5 & 2.03 \\
\hline ТPO & 0.06 & 0.005 & 0.75 & 0.06 \\
\hline TPO-L & 0.07 & 0.006 & 2.39 & 0.19 \\
\hline PI-819 & 0.02 & 0.001 & 0.61 & 0.05 \\
\hline$\sum$ P-PIs & 1.97 & 0.16 & 25.1 & 2.04 \\
\hline$\sum$ PIs & 11.4 & 0.93 & 86.9 & 7.06 \\
\hline
\end{tabular}

The estimated daily intake (EDI) was calculated from the equation as below:

$$
\mathrm{EDI}=\mathrm{C} \times \mathrm{DIR} / \mathrm{BW}
$$

Where " $\mathrm{C}$ " is is the concentration of target analyte, "DIR" is the dust ingestion rate (g/day), "BW" is the body weight $(\mathrm{kg})$. The body weights for toddlers and adults were 
13 and $80 \mathrm{~kg},{ }^{1}$ respectively, and "DIR" of 0.1 and $0.05 \mathrm{~g} / \mathrm{d}$ was used for toddlers and adults, ${ }^{1}$ respectively. 
Table S8. Descriptive statistics of the measured PI concentrations (ng/g) in different dust matrices.

Office dust ( $\mathrm{n}=62)$

Home dust ( $\mathrm{n}=30)$

\begin{tabular}{|c|c|c|c|c|c|c|}
\hline & $\mathrm{GM}^{\mathrm{a}}$ & Range & Quantification frequency (\%) & $\mathrm{GM}^{\mathrm{a}}$ & Range & Quantification frequency (\%) \\
\hline $\mathrm{BP}$ & 473 & $113-3131$ & 100 & 582 & $58.9-16670$ & 100 \\
\hline Benzil & 61.9 & $<\mathrm{MQL}-3521$ & 92 & 20.0 & $<\mathrm{MQL}-891$ & 80 \\
\hline PI-184 & 31.8 & $<\mathrm{MQL}-597$ & 82 & 211 & $8.75-6841$ & 100 \\
\hline PI-651 & 9.36 & $<\mathrm{MQL}-343$ & 90 & 21.5 & $<\mathrm{MQL}-150$ & 97 \\
\hline 4-MBP & 6.67 & $<\mathrm{MQL}-46.7$ & 94 & 16.0 & $2.32-116$ & 100 \\
\hline MBB & 19.7 & $\begin{array}{c}<\mathrm{MQL}- \\
10354\end{array}$ & 92 & 15.1 & $<\mathrm{MQL}-366$ & 90 \\
\hline PBZ & 6.55 & $<\mathrm{MQL}-1888$ & 97 & 10.1 & $<\mathrm{MQL}-116$ & 97 \\
\hline EAQ & 5.17 & $<\mathrm{MQL}-106$ & 3 & 5.12 & $<\mathrm{MQL}-54.9$ & 3 \\
\hline$\sum \mathrm{BZPs}$ & 772 & $136-13181$ & 100 & 1051 & $161-22122$ & 100 \\
\hline MK & 7.95 & $<\mathrm{MQL}-55.2$ & 98 & 2.71 & $<\mathrm{MQL}-12.9$ & 93 \\
\hline MEK & 9.46 & $<\mathrm{MQL}-95.1$ & 98 & 2.65 & $0.46-13.0$ & 100 \\
\hline $\mathrm{EAB}$ & 12.5 & $<\mathrm{MQL}-567$ & 77 & 4.90 & $<\mathrm{MQL}-109$ & 63 \\
\hline EDMAB & 4.15 & $<\mathrm{MQL}-1548$ & 76 & 1.88 & $<\mathrm{MQL}-15.4$ & 83 \\
\hline EHDAB & 9.42 & $<\mathrm{MQL}-1059$ & 97 & 3.67 & $<\mathrm{MQL}-979$ & 97 \\
\hline PI-369 & 0.81 & $<\mathrm{MQL}-107$ & 13 & 1.21 & $<\mathrm{MQL}-32.4$ & 30 \\
\hline
\end{tabular}




\begin{tabular}{|c|c|c|c|c|c|c|}
\hline PI-907 & 3.09 & $<\mathrm{MQL}-392$ & 58 & 3.15 & $<\mathrm{MQL}-72.4$ & 73 \\
\hline DABP & 3.20 & $<\mathrm{MQL}-58.0$ & 76 & 0.59 & $<\mathrm{MQL}-8.45$ & 63 \\
\hline DMAB & 8.01 & $<\mathrm{MQL}-74.3$ & 90 & 2.97 & $<\mathrm{MQL}-21.2$ & 93 \\
\hline$\sum \mathrm{ACIs}$ & 109 & $10.6-3231$ & 100 & 46.7 & $5.97-1001$ & 100 \\
\hline $\mathrm{TX}$ & 1.79 & $<\mathrm{MQL}-32.1$ & 8 & 1.61 & $<\mathrm{MQL}-3.82$ & 3 \\
\hline 2-ITX & 16.5 & $<\mathrm{MQL}-942$ & 92 & 13.1 & $0.25-399$ & 100 \\
\hline DETX & 2.11 & $<\mathrm{MQL}-508$ & 52 & 3.47 & $<\mathrm{MQL}-85.9$ & 87 \\
\hline 2-Cl-TX & $<\mathrm{MQL}$ & $<\mathrm{MQL}$ & 0 & $<\mathrm{MQL}$ & $<\mathrm{MQL}$ & 0 \\
\hline$\sum \mathrm{TXs}$ & 34.0 & $<\mathrm{MQL}-1451$ & 95 & 23.3 & $2.16-409$ & 100 \\
\hline ТРPO & 309 & $\begin{array}{c}<\mathrm{MQL}- \\
16318\end{array}$ & 98 & 52.6 & $<\mathrm{MQL}-525$ & 97 \\
\hline ТРО & 8.95 & $<\mathrm{MQL}-260$ & 55 & 7.15 & $<\mathrm{MQL}-123$ & 50 \\
\hline TPO-L & 6.64 & $<\mathrm{MQL}-197$ & 26 & 17.2 & $\begin{array}{c}<\mathrm{MQL}- \\
1022\end{array}$ & 43 \\
\hline PI-819 & 1.96 & $<\mathrm{MQL}-272$ & 11 & 1.98 & $<\mathrm{MQL}-208$ & 17 \\
\hline$\sum \mathrm{POs}$ & 370 & $\begin{array}{c}<\mathrm{MQL}- \\
16326\end{array}$ & 98 & 123 & $24.3-1183$ & 100 \\
\hline$\sum$ PIs & 1551 & $351-18067$ & 100 & 1352 & $204-23625$ & 100 \\
\hline
\end{tabular}


Table S9. Spearman correlation analysis of PIs in indoor dust samples $(\mathrm{n}=92)$.

\begin{tabular}{|c|c|c|c|c|c|c|c|c|c|c|c|c|c|c|c|c|c|c|c|}
\hline & MEK & PI-907 & EHDAB & MK & DMAB & EDMAB & EAB & DABP & PBZ & MBB & Benzil & PI-184 & $\mathrm{BP}$ & PI-651 & MBP & ТPO & ТРPO & 2-ITX & DETX \\
\hline MEK & 1 & & & & & & & & & & & & & & & & & & \\
\hline PI-907 & $.237^{*}$ & 1 & & & & & & & & & & & & & & & & & \\
\hline EHDAB & $0697 * *$ & $.213^{*}$ & 1 & & & & & & & & & & & & & & & & \\
\hline MK & $.836^{* *}$ & .107 & $.709 * *$ & 1 & & & & & & & & & & & & & & & \\
\hline DMAB & $.588^{* *}$ & $.238^{*}$ & $.545^{* *}$ & $.665 * *$ & 1 & & & & & & & & & & & & & & \\
\hline EDMAB & $.644 * *$ & $.307 * *$ & $.697 * *$ & $.638 * *$ & $.443 * *$ & 1 & & & & & & & & & & & & & \\
\hline EAB & .162 & .056 & $.208^{*}$ & .185 & $.371 * *$ & $.212^{*}$ & 1 & & & & & & & & & & & & \\
\hline DABP & $.326^{* *}$ & -.075 & $.271 * *$ & $.263^{*}$ & .100 & .174 & -.042 & 1 & & & & & & & & & & & \\
\hline PBZ & .015 & .117 & .041 & .048 & .181 & .132 & $.359^{* *}$ & -.178 & 1 & & & & & & & & & & \\
\hline MBB & .084 & .019 & $.266^{*}$ & .067 & .164 & $.348^{* *}$ & $.253 *$ & .012 & $.494 * *$ & 1 & & & & & & & & & \\
\hline Benzil & -.020 & -.094 & .049 & -.085 & .069 & .024 & .086 & .086 & .063 & $.249 *$ & 1 & & & & & & & & \\
\hline PI-184 & .040 & $.257^{*}$ & .185 & .113 & .171 & $.266^{*}$ & .061 & $-.363^{* *}$ & $.411^{* *}$ & $.403 * *$ & -.140 & 1 & & & & & & & \\
\hline BP & $.230^{*}$ & $.265^{*}$ & $.413 * *$ & $.242 *$ & $.334 * *$ & $.412 * *$ & .131 & -.111 & $.294 * *$ & $.469^{* *}$ & $.236^{* *}$ & $.566^{* *}$ & 1 & & & & & & \\
\hline PI-651 & .008 & $.248^{*}$ & .170 & .066 & .033 & $.242 *$ & .081 & $-.235^{*}$ & $.365^{*}$ & $.293 * *$ & .011 & $.545^{* *}$ & $.420^{* *}$ & 1 & & & & & \\
\hline MBP & $-.415^{* *}$ & -.003 & $-.261^{*}$ & $-.413 * *$ & $-.342 * *$ & $-.319 * *$ & -.027 & -.109 & .101 & .101 & .160 & $.310^{* * *}$ & .103 & $.249 *$ & 1 & & & & \\
\hline ТPO & $.366^{* *}$ & $.216^{*}$ & $.321 * *$ & $.431 * *$ & $.505 * *$ & $.428 * *$ & $.213^{*}$ & -.199 & $.371^{* *}$ & $.258^{*}$ & .005 & $.438^{* *}$ & .439 ** & $.378^{* *}$ & -.094 & 1 & & & \\
\hline ТРPО & $.358^{* *}$ & .000 & $.302 * *$ & $.306 * *$ & $.428^{* *}$ & $.255^{*}$ & .174 & .146 & $.220^{*}$ & $.234 *$ & $.518^{* *}$ & -.161 & .167 & -.021 & -.146 & .178 & 1 & & \\
\hline 2-ITX & $.690^{* *}$ & $.291 * *$ & $.762 * *$ & $.735^{* *}$ & $.494 * *$ & $.692 * *$ & .196 & .103 & $.328 * *$ & $.294 * *$ & -.033 & $.334^{* *}$ & $.413^{* *}$ & $.300^{* *}$ & $-.260^{*}$ & $.469^{* *}$ & $.362 * *$ & 1 & \\
\hline DETX & $.346^{* *}$ & $.403^{* *}$ & $.445 * *$ & $.372 * *$ & $.449 * *$ & $.463 * *$ & $.291 * *$ & $-.258^{*}$ & $.380^{* *}$ & $.226^{*}$ & .030 & $.439^{* *}$ & $.459^{* *}$ & $.423^{* *}$ & -.118 & $.659^{* *}$ & $.213^{*}$ & $.609 * *$ & 1 \\
\hline
\end{tabular}


Table S10. The starting concentrations and calculated half-life times $\left(t_{1 / 2}\right)$ of PIs in food packaging materials used for the photodegradation.

\begin{tabular}{|c|c|c|}
\hline Compounds & $\begin{array}{l}\text { Mean } \pm \text { standard deviation } \\
\qquad\left(\mathrm{n}=3, \mathrm{ng} / \mathrm{dm}^{2}\right)\end{array}$ & $\mathrm{t}_{1 / 2}(\mathrm{~h})$ \\
\hline $\mathrm{BP}$ & $23049 \pm 749$ & 248 \\
\hline Benzil & $6.50 \pm 1.08$ & $-\mathrm{a}$ \\
\hline PI-184 & $1355 \pm 57.0$ & 99 \\
\hline PI-651 & $940 \pm 162.9$ & 42 \\
\hline 4-MBP & $1003 \pm 71.4$ & 82 \\
\hline MBB & $2849 \pm 160$ & 144 \\
\hline PBZ & $2004 \pm 38.2$ & 147 \\
\hline EAQ & $<\mathrm{MQL}$ & $-{ }^{\mathrm{a}}$ \\
\hline MK & $432 \pm 15.5$ & 75 \\
\hline MEK & $181 \pm 7.88$ & 40 \\
\hline $\mathrm{EAB}$ & $36.8 \pm 2.19$ & $-{ }^{a}$ \\
\hline EDMAB & $2739 \pm 152$ & 173 \\
\hline EHDAB & $1410 \pm 74.1$ & 34 \\
\hline PI-369 & $404 \pm 44.4$ & 48 \\
\hline PI-907 & $15.4 \pm 1.61$ & 41 \\
\hline DABP & $1.20 \pm 0.07$ & $-{ }^{\mathrm{a}}$ \\
\hline DMAB & $38.9 \pm 2.31$ & 82 \\
\hline $\mathrm{TX}$ & $<\mathrm{MQL}$ & $-\mathrm{b}$ \\
\hline 2-ITX & $261 \pm 6.92$ & 61 \\
\hline DETX & $324 \pm 7.17$ & 94 \\
\hline 2-Cl-TX & $<\mathrm{MQL}$ & $-\mathrm{b}$ \\
\hline ТРPO & $168 \pm 15.4$ & 289 \\
\hline TPO & $299 \pm 17.2$ & 50 \\
\hline TPO-L & $226 \pm 19.2$ & 53 \\
\hline PI-819 & $30.6 \pm 5.97$ & 32 \\
\hline
\end{tabular}


Table S11. Ratios between the measured concentrations of transformation products and parent chemicals.

\begin{tabular}{llccc}
\hline \multicolumn{1}{c}{ Ratios } & GM & median & range \\
& \multicolumn{1}{c}{ 2-ITX-O/2-ITX } & 0.09 & 0.090 & $0.021-0.31$ \\
Food & 2-ITX-O $2 / 2-I T X$ & 0.06 & 0.057 & $0.0028-2.22$ \\
materials & DETX-O/DETX & 0.08 & 0.092 & $0.0091-1.33$ \\
$(\mathrm{n}=33)$ & DETX-O $_{2} /$ DETX & 0.03 & 0.013 & $0.0007-14.8$ \\
& 2-ITX-O/2-ITX & 0.35 & 0.98 & $0.0046-7.19$ \\
Office dust & 2-ITX-O $/ 2-I T X$ & 0.70 & 6.90 & $0.0087-227$ \\
$(\mathrm{n}=92)$ & DETX-O/DETX & 1.05 & 15.1 & $0.0035-202$ \\
& DETX-O $_{2} /$ DETX & 1.00 & 28.8 & $0.0055-796$ \\
\hline
\end{tabular}


Table S12. Human skin sensitization predicted by Pre-Skin.

\begin{tabular}{|c|c|c|c|}
\hline Compounds & SMILES & Result & $\begin{array}{l}\text { Probability } \\
\quad(\%)\end{array}$ \\
\hline BP & $\mathrm{O}=\mathrm{C}(\mathrm{c}(\operatorname{ccc} 1) \mathrm{c} 1) \mathrm{c}(\operatorname{ccc} 2) \mathrm{c} 2$ & Sensitizer & 70 \\
\hline Benzil & $\mathrm{O}=\mathrm{C}(\mathrm{c}(\operatorname{ccc} 1) \mathrm{c} 1) \mathrm{C}(=\mathrm{O}) \mathrm{c}(\operatorname{ccc} 2) \mathrm{c} 2$ & Sensitizer & 70 \\
\hline PI-184 & $\mathrm{O}=\mathrm{C}(\mathrm{c}(\mathrm{cccc} 1) \mathrm{c} 1) \mathrm{C}(\mathrm{O})(\mathrm{CCCC} 2) \mathrm{C} 2$ & Non-sensitizer & 50 \\
\hline 4-MBP & $\mathrm{O}=\mathrm{C}(\mathrm{c}(\operatorname{ccc} 1) \mathrm{c} 1) \mathrm{c}(\operatorname{ccc}(\mathrm{c} 2) \mathrm{C}) \mathrm{c} 2$ & Non-sensitizer & 50 \\
\hline EAQ & $\mathrm{O}=\mathrm{C}(\mathrm{c}(\mathrm{c}(\mathrm{C}(=\mathrm{O}) \mathrm{c} 1 \mathrm{ccc} 2) \operatorname{ccc} 3 \mathrm{CC}) \mathrm{c} 3) \mathrm{c} 12$ & Sensitizer & 80 \\
\hline PBZ & $\mathrm{O}=\mathrm{C}(\mathrm{c}(\operatorname{ccc}(\mathrm{c}(\operatorname{ccc} 1) \mathrm{c} 1) \mathrm{c} 2) \mathrm{c} 2) \mathrm{c}(\operatorname{ccc} 3) \mathrm{c} 3$ & Sensitizer & 60 \\
\hline PI-651 & $\mathrm{O}=\mathrm{C}(\mathrm{c}(\mathrm{cccc} 1) \mathrm{c} 1) \mathrm{C}(\mathrm{OC})(\mathrm{OC}) \mathrm{c}(\operatorname{ccc} 2) \mathrm{c} 2$ & Non-sensitizer & 50 \\
\hline MBPPS & $\mathrm{Cc} 1 \operatorname{ccc}(\operatorname{cc} 1) \operatorname{Sc} 2 \operatorname{ccc}(\operatorname{cc} 2) \mathrm{C}(=\mathrm{O}) \operatorname{c} 3 \operatorname{ccc} c 3$ & Sensitizer & 60 \\
\hline MBB & $\mathrm{O}=\mathrm{C}(\mathrm{OC}) \mathrm{c}(\mathrm{c}(\mathrm{ccc} 1) \mathrm{C}(=\mathrm{O}) \mathrm{c}(\operatorname{ccc} 2) \mathrm{c} 2) \mathrm{c} 1$ & Non-sensitizer & 70 \\
\hline MK & $\mathrm{O}=\mathrm{C}(\mathrm{c}(\operatorname{ccc}(\mathrm{N}(\mathrm{C}) \mathrm{C}) \mathrm{c} 1) \mathrm{c} 1) \mathrm{c}(\operatorname{ccc}(\mathrm{N}(\mathrm{C}) \mathrm{C}) \mathrm{c} 2) \mathrm{c} 2$ & Sensitizer & 60 \\
\hline MEK & $\begin{array}{l}\mathrm{O}=\mathrm{C}(\mathrm{c}(\operatorname{ccc}(\mathrm{N}(\mathrm{CC}) \mathrm{CC}) \mathrm{c} 1) \mathrm{c} 1) \mathrm{c}(\mathrm{ccc}(\mathrm{N}(\mathrm{CC}) \mathrm{CC}) \\
\mathrm{c} 2) \mathrm{c} 2\end{array}$ & Sensitizer & 60 \\
\hline EDMAB & $\mathrm{O}=\mathrm{C}(\mathrm{OCC}) \mathrm{c}(\operatorname{ccc}(\mathrm{N}(\mathrm{C}) \mathrm{C}) \mathrm{c} 1) \mathrm{c} 1$ & Sensitizer & 70 \\
\hline EHDAB & $\mathrm{O}=\mathrm{C}(\mathrm{OCC}(\mathrm{CCCC}) \mathrm{CC}) \mathrm{c}(\operatorname{ccc}(\mathrm{N}(\mathrm{C}) \mathrm{C}) \mathrm{c} 1) \mathrm{c} 1$ & Sensitizer & 80 \\
\hline PI-369 & $\begin{array}{l}\mathrm{CCC}(\mathrm{C}(=\mathrm{O}) \operatorname{c} 1 \operatorname{ccc}(\mathrm{cc} 1) \mathrm{N} 2 \mathrm{CCOCC} 2)(\mathrm{N}(\mathrm{C}) \mathrm{C}) \\
\mathrm{Cc} 3 \operatorname{ccccc} 3\end{array}$ & Non-sensitizer & 50 \\
\hline PI-907 & $\mathrm{O}=\mathrm{C}(\mathrm{c} 1 \mathrm{ccc}(\mathrm{cc} 1) \mathrm{SC}) \mathrm{C}(\mathrm{N} 2 \mathrm{CCOCC} 2)(\mathrm{C}) \mathrm{C}$ & Non-sensitizer & 50 \\
\hline DMAB & $\mathrm{O}=\mathrm{C}(\mathrm{c}(\operatorname{ccc}(\mathrm{N}(\mathrm{C}) \mathrm{C}) \mathrm{c} 1) \mathrm{c} 1) \mathrm{c}(\operatorname{ccc} 2) \mathrm{c} 2$ & Sensitizer & 60 \\
\hline EAB & $\mathrm{O}=\mathrm{C}(\mathrm{OCC}) \mathrm{c}(\operatorname{ccc}(\mathrm{N}) \mathrm{c} 1) \mathrm{c} 1$ & Sensitizer & 60 \\
\hline $\mathrm{TX}$ & $\operatorname{c} 1 \operatorname{ccc} 2 \mathrm{Sc} 3 \operatorname{ccc} c 3 \mathrm{C}(=\mathrm{O}) \mathrm{c} 2 \mathrm{c} 1$ & Sensitizer & 80 \\
\hline 2-ITX & $\operatorname{c} 1 \operatorname{cc} 2 \mathrm{Sc} 3 \operatorname{ccc}(\mathrm{C}(\mathrm{C}) \mathrm{C}) \operatorname{cc} 3 \mathrm{C}(=\mathrm{O}) \mathrm{c} 2 \mathrm{cc} 1$ & Sensitizer & 80 \\
\hline 2-Cl-TX & $\mathrm{c} 12 \mathrm{C}(=\mathrm{O}) \mathrm{c} 3 \mathrm{c}(\operatorname{ccc}(\mathrm{Cl}) \mathrm{c} 3) \mathrm{Sc} 1 \mathrm{ccc} 2$ & Sensitizer & 90 \\
\hline DETX & $\mathrm{c} 12 \mathrm{C}(=\mathrm{O}) \mathrm{c} 3 \mathrm{c}(\mathrm{c}(\mathrm{CC}) \mathrm{cc}(\mathrm{CC}) \mathrm{c} 3) \mathrm{Sc} 1 \mathrm{ccc} 2$ & Sensitizer & 70 \\
\hline 2-ITX-O & $\mathrm{CC}(\mathrm{c} 1 \mathrm{ccc} 2 \mathrm{c}(\mathrm{c} 1) \mathrm{c}(=\mathrm{O}) \mathrm{c} 1 \mathrm{c}(\mathrm{s} 2=\mathrm{O}) \mathrm{ccc} 1) \mathrm{C}$ & Sensitizer & 60 \\
\hline $2-\mathrm{ITX}-\mathrm{O}_{2}$ & $\mathrm{O}=\mathrm{C} 1 \mathrm{c} 2 \operatorname{cc}(\operatorname{ccc} 2 \mathrm{~S}(=\mathrm{O})(=\mathrm{O}) \mathrm{c} 2 \mathrm{c} 1 \operatorname{ccc} 2) \mathrm{C}(\mathrm{C}) \mathrm{C}$ & Sensitizer & 60 \\
\hline DETX-O & $\mathrm{CCc} 1 \mathrm{cc}(\mathrm{CC}) \mathrm{cc} 2 \mathrm{c} 1 \mathrm{~s}(=\mathrm{O}) \mathrm{c} 1 \mathrm{c}(\mathrm{c} 2=\mathrm{O}) \mathrm{ccc} 1$ & Non-sensitizer & 50 \\
\hline DETX-O ${ }_{2}$ & $\begin{array}{l}\operatorname{CCc} 1 \mathrm{cc}(\mathrm{CC}) \operatorname{cc} 2 \mathrm{c} 1 \mathrm{~S}(=\mathrm{O})(=\mathrm{O}) \mathrm{c} 1 \mathrm{c}(\mathrm{C} 2=\mathrm{O}) \operatorname{cccc} \\
1\end{array}$ & Non-sensitizer & 50 \\
\hline ТРPO & $\mathrm{O}=\mathrm{P}(\mathrm{c} 1 \mathrm{cccc} 1)(\mathrm{c} 1 \operatorname{ccc} \mathrm{c} 1) \mathrm{c} 1 \mathrm{cccc} 1$ & Sensitizer & 70 \\
\hline TPO & $\begin{array}{l}\operatorname{Cc} 1 \operatorname{cc}(\mathrm{C}) \mathrm{c}(\mathrm{c}(\mathrm{c} 1) \mathrm{C}) \mathrm{C}(=\mathrm{O}) \mathrm{P}(=\mathrm{O})(\mathrm{c} 1 \operatorname{cccc} 1) \mathrm{c} 1 \mathrm{c} \\
\operatorname{ccc} 1\end{array}$ & Sensitizer & 60 \\
\hline TPO-L & $\operatorname{CCOP}(=\mathrm{O})(\mathrm{C}(=\mathrm{O}) \mathrm{c} 1 \mathrm{c}(\mathrm{C}) \operatorname{cc}(\operatorname{cc} 1 \mathrm{C}) \mathrm{C}) \mathrm{c} 1 \mathrm{ccccc} 1$ & Non-sensitizer & 50 \\
\hline PI-819 & $\begin{array}{l}\mathrm{Cc} 1 \mathrm{cc}(\mathrm{C}) \mathrm{c}(\mathrm{c}(\mathrm{c} 1) \mathrm{C}) \mathrm{C}(=\mathrm{O}) \mathrm{P}(=\mathrm{O})(\mathrm{C}(=\mathrm{O}) \mathrm{c} 1 \mathrm{c}(\mathrm{C}) \\
\operatorname{cc}(\operatorname{cc} 1 \mathrm{C}) \mathrm{C}) \mathrm{c} 1 \mathrm{cccc} 1\end{array}$ & Sensitizer & 60 \\
\hline
\end{tabular}


Figure S1. Structures of the target BZPs, TXs, and ACIs in the present study.

\section{Benzophenones (BZPs)}<smiles>O=C(c1ccccc1)c1ccccc1</smiles>

BP<smiles>CCc1ccc2c(c1)C(=O)c1ccccc1C2=O</smiles>

EAQ<smiles>Cc1ccc(C(=O)c2ccccc2)cc1</smiles>

4-MBP<smiles>COC(=O)c1ccccc1C(=O)c1ccccc1</smiles>

MBB<smiles>O=C(c1ccccc1)C1(O)CCCCC1</smiles>

PI-184<smiles>COC(OC)(c1ccccc1)c1ccccc1</smiles>

PI-651<smiles>O=C(C(=O)c1ccccc1)c1ccccc1</smiles>

Benzil

Thioxanthones (TXs)<smiles>O=c1c2ccccc2sc2ccccc12</smiles>

TX<smiles>CC(C)c1ccc2sc3ccccc3c(=O)c2c1</smiles>

2-ITX<smiles>CCc1cc(CC)c2sc3ccccc3c(=O)c2c1</smiles>

DETX<smiles>O=C(c1ccccc1)c1ccc(-c2ccccc2)cc1</smiles>

PBZ<smiles>O=c1c2ccccc2sc2ccc(Cl)cc12</smiles>

2-Cl-TX

Amine co-initiators (ACIs)<smiles>CN(C)c1ccc(C(=O)c2ccccc2)cc1</smiles><smiles>CN(C)c1ccc(C(=O)c2ccc(N(C)C)cc2)cc1</smiles><smiles>CCN(CC)c1ccc(C(=O)c2ccc(N(CC)CC)cc2)cc1</smiles><smiles>CSc1ccc(C(=O)C(C)(C)N2CCOCC2)cc1</smiles>

DMAB

MK

MEK

PI-907<smiles>CCOC(=O)c1ccc(N)cc1</smiles>

EAB<smiles>CCOC(=O)c1ccc(N(C)C)cc1</smiles>

EDMAB<smiles>CCCCC(CC)COC(=O)c1ccc(N(C)C)cc1</smiles>

EHDAB<smiles>CCC(Cc1ccccc1)(Cc1ccc(N2CCOCC2)cc1)C(=O)c1ccccc1</smiles>

PI-369<smiles>Nc1ccc(C(=O)c2ccc(N)cc2)cc1</smiles> 
Figure S2. Stability of PIs in food packaging materials in the dark $(n=3)$.
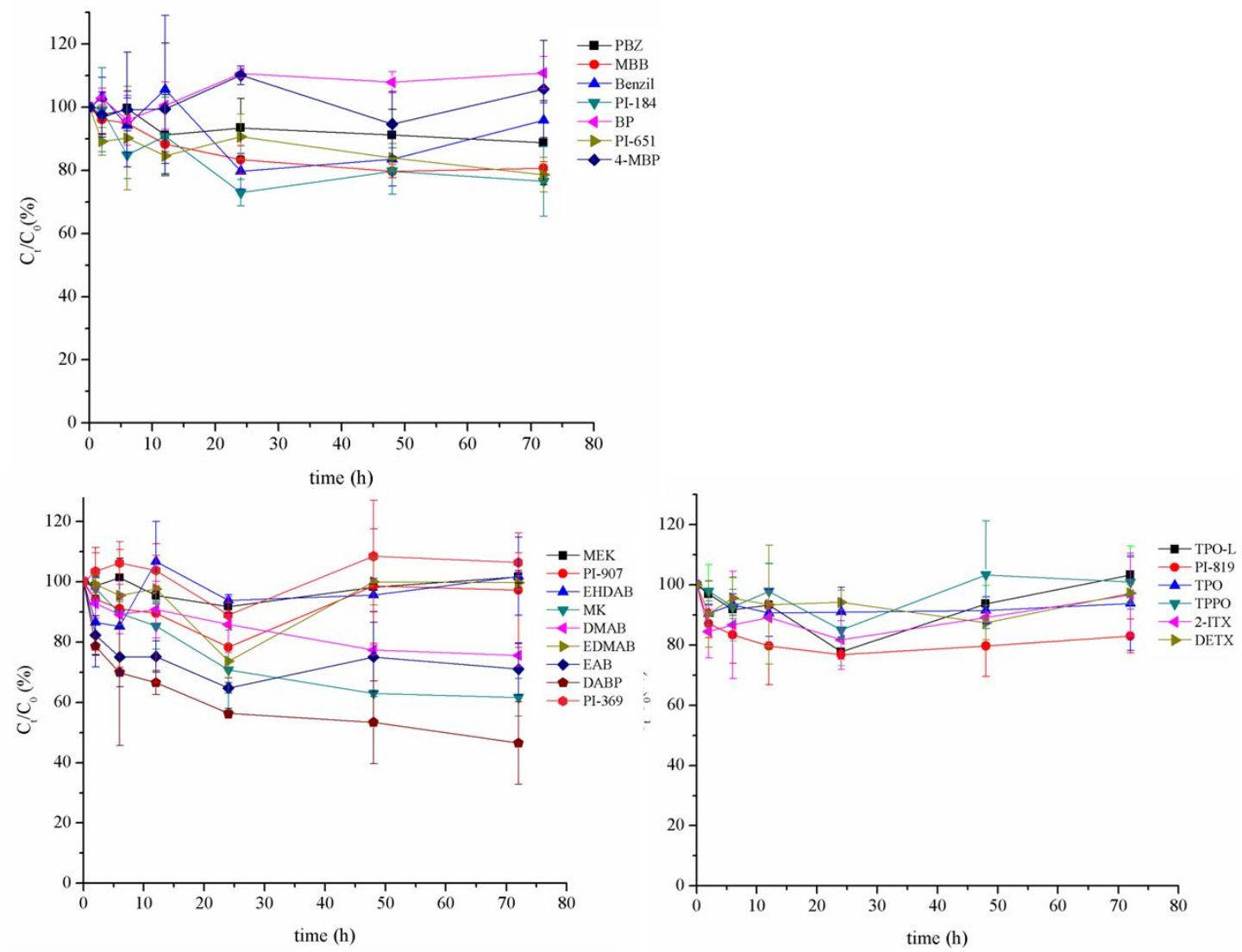
Figure S3. Concentrations of TX transformation products (mean \pm standard deviation, $\mathrm{n}=3$ ) in food packaging materials during the photodegradation experiments.

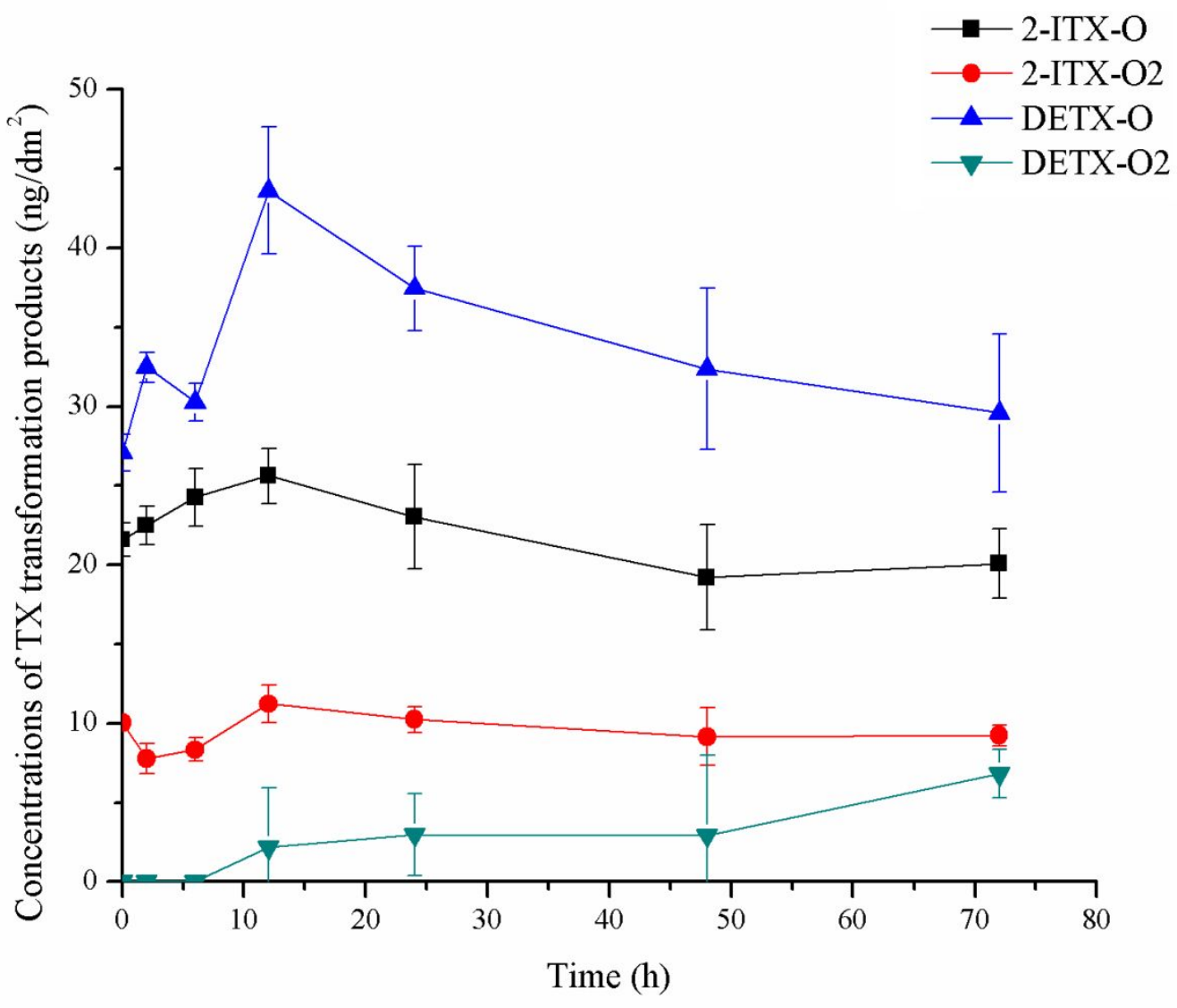

\section{Reference:}

1. Zheng, X. B.; Qiao, L.; Covaci, A.; Sun, R. X.; Guo, H. Y.; Zheng, J.; Luo, X. J.; Xie, Q. L.; Mai, B. X., Brominated and phosphate flame retardants (FRs) in indoor dust from different microenvironments: Implications for human exposure via dust ingestion and dermal contact. Chemosphere 2017, 184, 185-191. 